\title{
Fatal Infectious Mononucleosis with Liver Failure in Two Sisters
}

\author{
J. T. HARRIES and A. W. FERGUSON \\ From The Hospital for Sick Children, Great Ormond Street, London W.C.1, and \\ Bedford General Hospital, Bedford
}

The purpose of this paper is to report an instance of liver failure in a fatal case of infectious mononucleosis in a child. Her younger sister had also died two months earlier from an acute illness originally though to be acute leukaemia; in retrospect the similarities of the illnesses in the two sisters suggested that both had suffered from the same infection.

\section{Case Reports}

Case 1. A girl aged 22 months was admitted to hospital with a history of swelling of the cervical glands on the right side of a few days' duration. There had been no response to oral penicillin, and the swelling had spread to the right side of the face. Both parents were well, the father Austrian and the mother British; there was a healthy brother aged 4 years. Only five weeks previously, a sister (Case 2) aged 3 months had died in the same hospital after a brief illness which had been thought to be acute leukaemia.

She was a moderately ill girl with a fever and considerable swelling of the cervical, pre-auricular, and post-auricular lymph nodes, and this was associated with an acute exudative tonsillitis. The spleen and liver were not enlarged. She was treated with penicillin but the fever continued, she became jaundiced, and enlargement of the liver ( 3 finger breadths) and spleen ( 1 finger breadth) appeared. Persistently loose stools led to slight dehydration, and she became listless and semicomatose with some head retraction and neck stiffness. 25 days after the onset of the illness, a fine pink macular rash, which was most marked on the trunk, covered the whole body, the lips were crusted and haemorrhagic, and infected ulcerative lesions involved the vulva, perineum, and buttocks. There were similar lesions on the buccal mucosa and soft palate, and pitting oedema was present in both thighs. The right submandibular, tonsillar, and upper cervical lymph nodes were much enlarged and matted together, while the other nodes were discrete and not greatly enlarged.

Table I shows investigations in relation to the day of illness. Other investigations performed during the terminal phase of the illness were as follows.

Received March 8, 1968.
Bone-marrow was moderately cellular, with normoblastic erythropoiesis; mononuclear cells were increased, many showing atypical features as seen in infectious mononucleosis; there was no increase in primitive cells. Some of the mononuclear cells were excessively vacuolated, and material within the vacuoles did not stain for glycogen or lipid.

Direct Coombs test negative. Total serum proteins $3.0 \mathrm{~g} . / 100 \mathrm{ml}$., and electrophoretic strip showed all fractions to be reduced except $\gamma$-globulin which was within normal limits. Partial thromboplastin time $>\mathbf{3 0 0}$ seconds (control 34.6 seconds); blood fibrinogen titre nil (control $1: 60$ ). Cytomegalovirus complementfixing antibody titre nil. Stool for occult blood positive. Urine: protein trace, moderate numbers of epethelial cells and bacteria, and culture showed a heavy growth of proteus and Strep. faecalis; bile present, no excess urobilinogen. Chest $x$-ray, ECG, ESR, and blood culture normal.

She was considered to be suffering from hepatic coma, secondary to liver damage caused by infectious mononucleosis, and was treated with a high carbohydrate, low protein diet, vitamin $K$, and neomycin, and over the next 48 hours her condition remained unchanged. She developed a metabolic acidosis which was corrected, and she was also given large doses of intravenous hydrocortisone, and a transient improvement in her level of consciousness followed. Frequent malaena stools resulted in her becoming anaemic and a thrombocytopenia was also noted; she was treated with blood and platelet transfusions. Some reduction in the size of the liver was noted, but she died 28 days after the onset of the illness.

Necropsy (18 hours after death). The stomach and intestine contained flecks of altered blood, and the oesophagus showed multiple perforations in the left postero-lateral region, but there was no reaction in the left chest suggesting that this was an agonal perforation. The liver weighed 524 g. (normal for age 394 g.), was soft and flabby to the touch, and was yellowish in colour. When cut, there were areas of necrosis which were mainly subscapular in position. The lungs were haemorrhagic and oedematous, and consolidation was present at both bases. The spleen was congested and weighed $85 \mathrm{~g}$. Lymph node enlargement was noted in 
TABLE I

Case 1: Blood Findings and Electroencephalogram

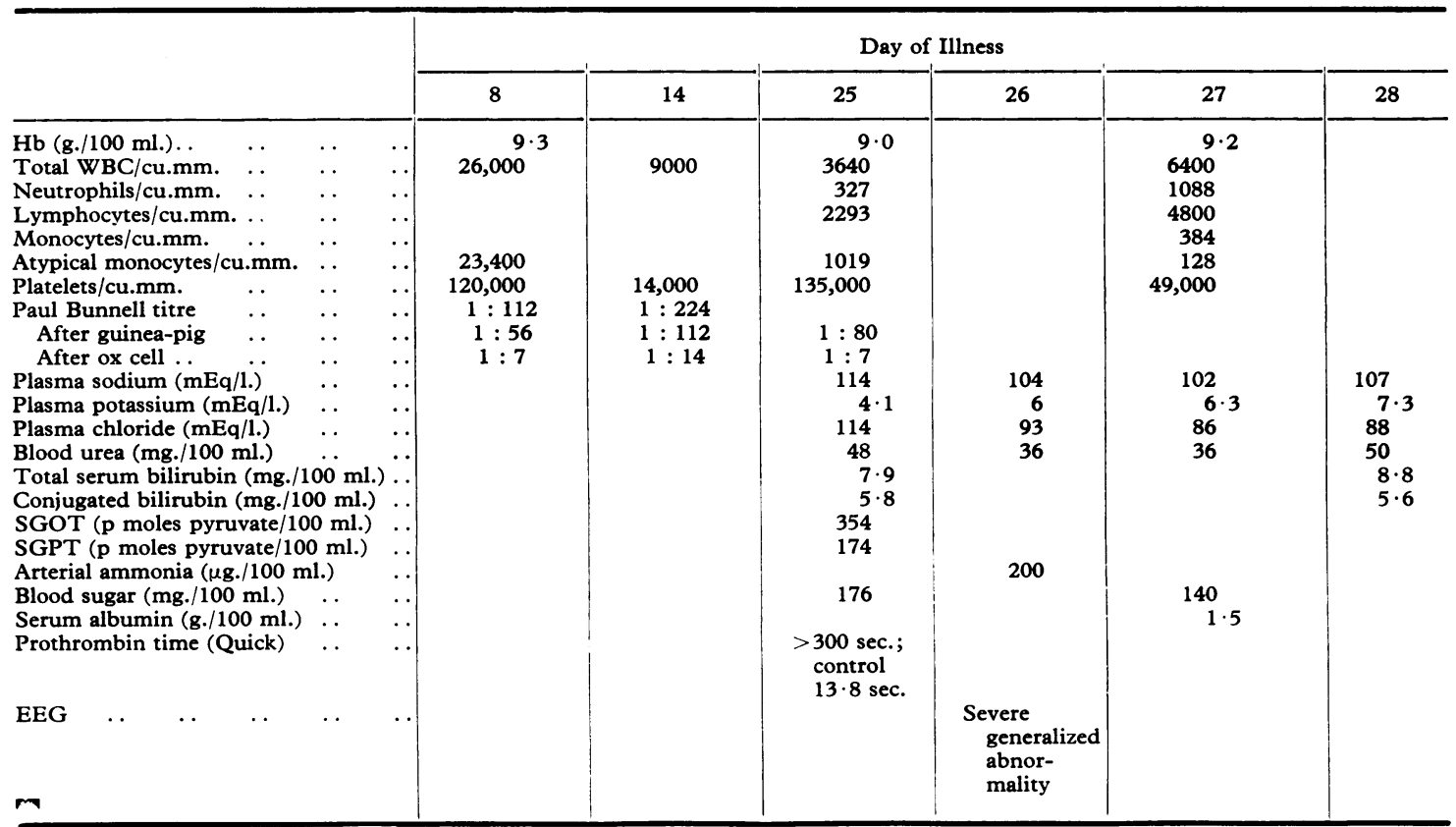

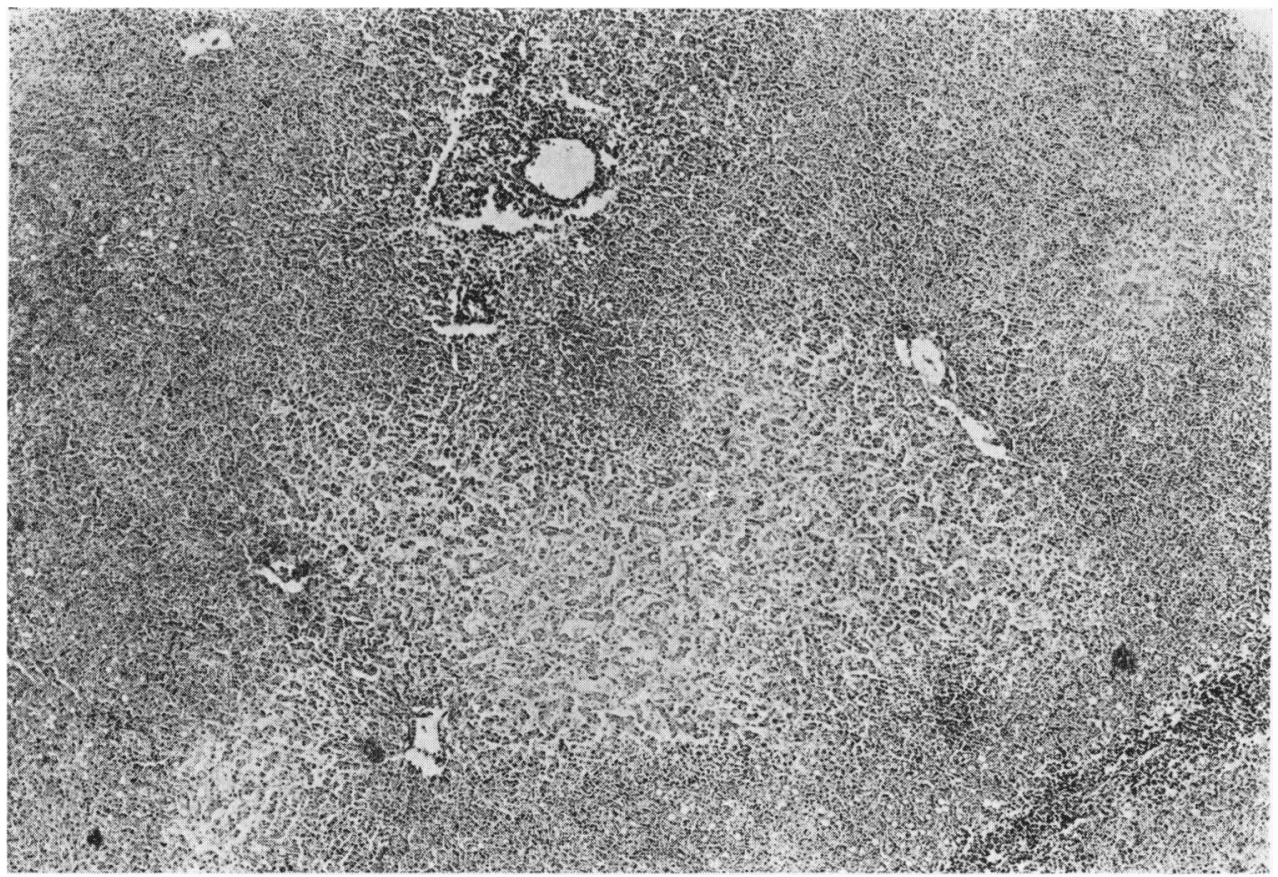

Fig. 1.-Case 1. Liver showing areas of necrosis and cellular infiltration. (H. and E. $\times 17$. 


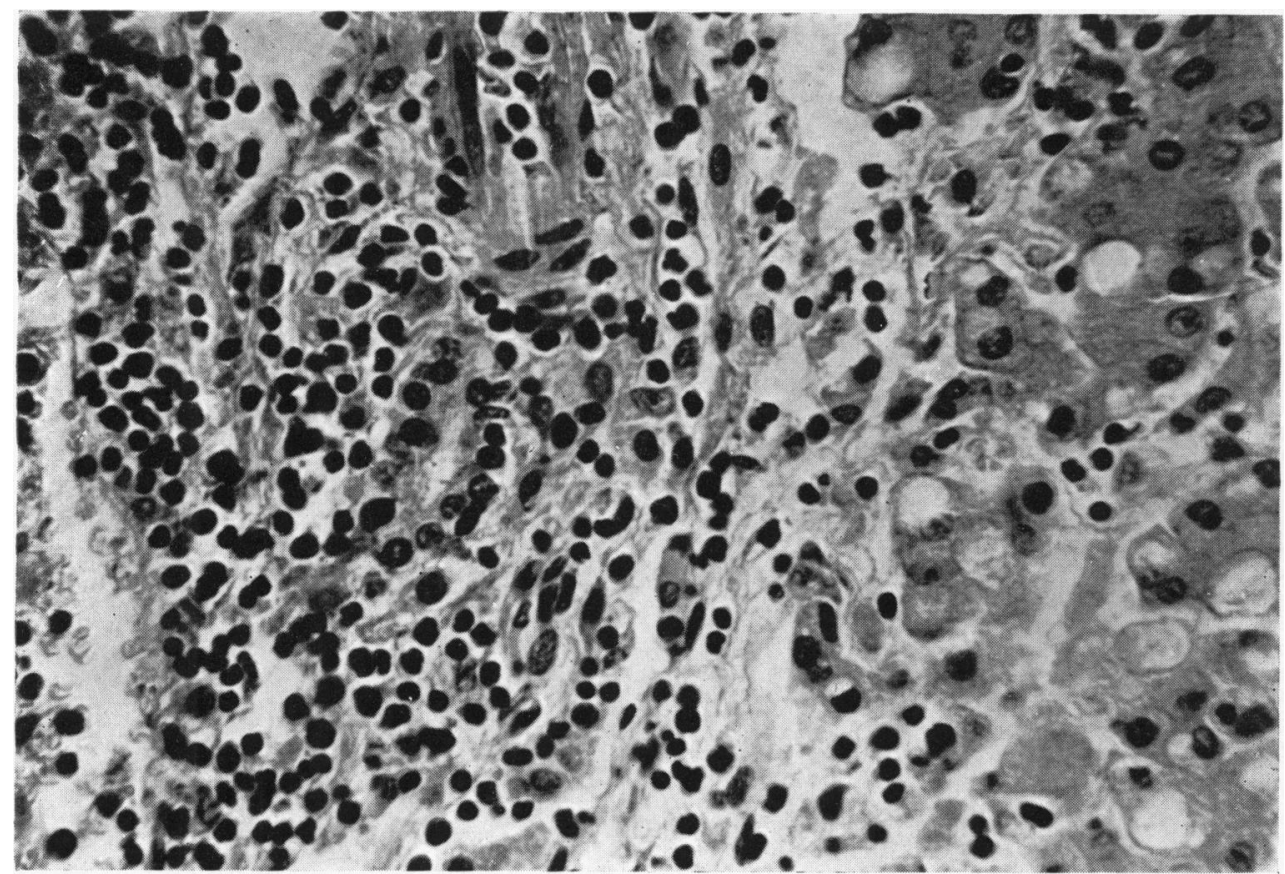

Fig. 2.-Case 1. Liver, showing mononuclear cell infiltrate in portal area. (H. and E. $\times 175$.

all the upper deep cervical and in some of the axillary and inguinal groups.

Histology. Liver (see Fig. 1 and 2). There was extensive mononuclear cell infiltration around the portal tracts, and occasional areas of focal hepatic necrosis were seen. Many parenchymal cells showed evidence of fatty degeneration, and there was some giant cell formation. The sinusoids were wide suggesting atrophy, and areas of more extensive necrosis were seen under the capsule.

Extensive mononuclear cell infiltration was seen in many organs including the pancreas, lymph nodes, adrenals, myocardium, spleen, kidneys, and lungs.

Case 2. The younger sister of Case 1 was born at term after a normal pregnancy, and weighed $2.36 \mathrm{~kg}$. She was well until 10 weeks old, when she developed a mild febrile illness associated with an erythematous rash. 2 weeks later, because of pallor and diarrhoea, she was admitted to hospital where she was found to be very ill, pale, and febrile $\left(38 \cdot 3^{\circ} \mathrm{C}\right.$.). The liver and spleen were both enlarged 3 finger breadths below the costal margin, and there was purpura, but no lymph node enlargement or jaundice. Table II shows the investigations performed. In addition urine examination showed 4 WBC per high-power field, no red cells or casts, and a heavy growth of Esch. coli. Stool examination for pathogens and occult blood was negative; throat swab grew no significant organisms; $x$-ray examination of skull, long bones, and chest was normal.

Bone-marrow, examined on the 17th day of illness, was very hypocellular; the main cells were lymphocytes and myelocytes. A small number of immature blastic leucocytes were seen. Erythropoiesis and megakaryocytopoiesis appeared virtually absent.

The most likely diagnosis was considered to be a leukaemia of some type, and she was treated with blood transfusions, prednisolone, and antibiotics. 4 days after admission an erythematous rash appeared, and enlargement of the posterior cervical, axillary, and inguinal lymph nodes was noted. The liver and spleen continued to enlarge, haematemesis occurred, and she died 10 days after admission.

Necropsy (21 hours after death). Bone-marrow from the sternum, lumbar, and thoracic vertebrae was macroscopically normal. All lymph nodes were slightly enlarged. The liver was enlarged $(325 \mathrm{~g}$.) and when cut showed a pale, reddish-brown surface with fine grey reticulation. The spleen was greatly enlarged (107 g.), and on cutting showed a purple red surface of uniform appearance. In the heart there were a few epicardial and subendocardial petechiae on the left ventricular surface of the interventricular septum. The left lung contained blotchy areas of haemorrhage, and the right lung had a similar appearance in the upper and middle lobes. The right lower lobe was deep red-grey, felt 
TABLE II

Case 2: Blood Findings

\begin{tabular}{|c|c|c|c|c|c|c|c|}
\hline & & \multicolumn{6}{|c|}{ Day of Illness } \\
\hline & & 14 & 15 & 16 & 17 & 21 & 23 \\
\hline $\begin{array}{l}\text { Hb (g./100 ml.).. } \\
\text { Total WBC/cu.mm. } \\
\text { Neutrophils/cu.mm. } \\
\text { Lymphocytes/cu.mm. } \\
\text { Atypical mononuclear } \\
\text { Platelets/cu.mm. } \\
\text { Paul Bunnell } \\
\text { Total serum bilirubin } \\
\text { Blood urea (mg./100 m } \\
\text { Toxoplasma dye test }\end{array}$ & 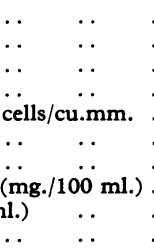 & $\begin{array}{c}4 \cdot 4 \\
4300 \\
175 \\
1800 \\
2200 \\
11,000 \\
0 \cdot 4 \\
22\end{array}$ & Negative & $11 \cdot 4$ & $\begin{array}{l}\text { Negative } \\
\text { Negative }\end{array}$ & $2200^{7 \cdot 8}$ & $2400^{5 \cdot 7}$ \\
\hline
\end{tabular}

very firm, and suggested a massive haemorrhage or infarction. The kidneys were slightly enlarged and pale. The gastro-intestinal tract, suprarenals, thyroid, cranial cavity, and brain were normal.

Histology. A rib showed small collections of abnormal mononuclear cells throughout the marrow. In the liver (see Fig. 3 and 4) there was extensive focal mononuclear cell infiltration of the portal tracts, and there was foamy swelling of the Kupffer cells. Extensive mononuclear cell infiltration was present in the heart, lungs, pancreas, and spleen.

\section{Discussion}

In a review of fatal cases of infectious mononucleosis, Custer and Smith (1948) found that infiltration by typical and atypical lymphocytes was very common in the interstitial tissues and blood vessel walls of most organs. Similar

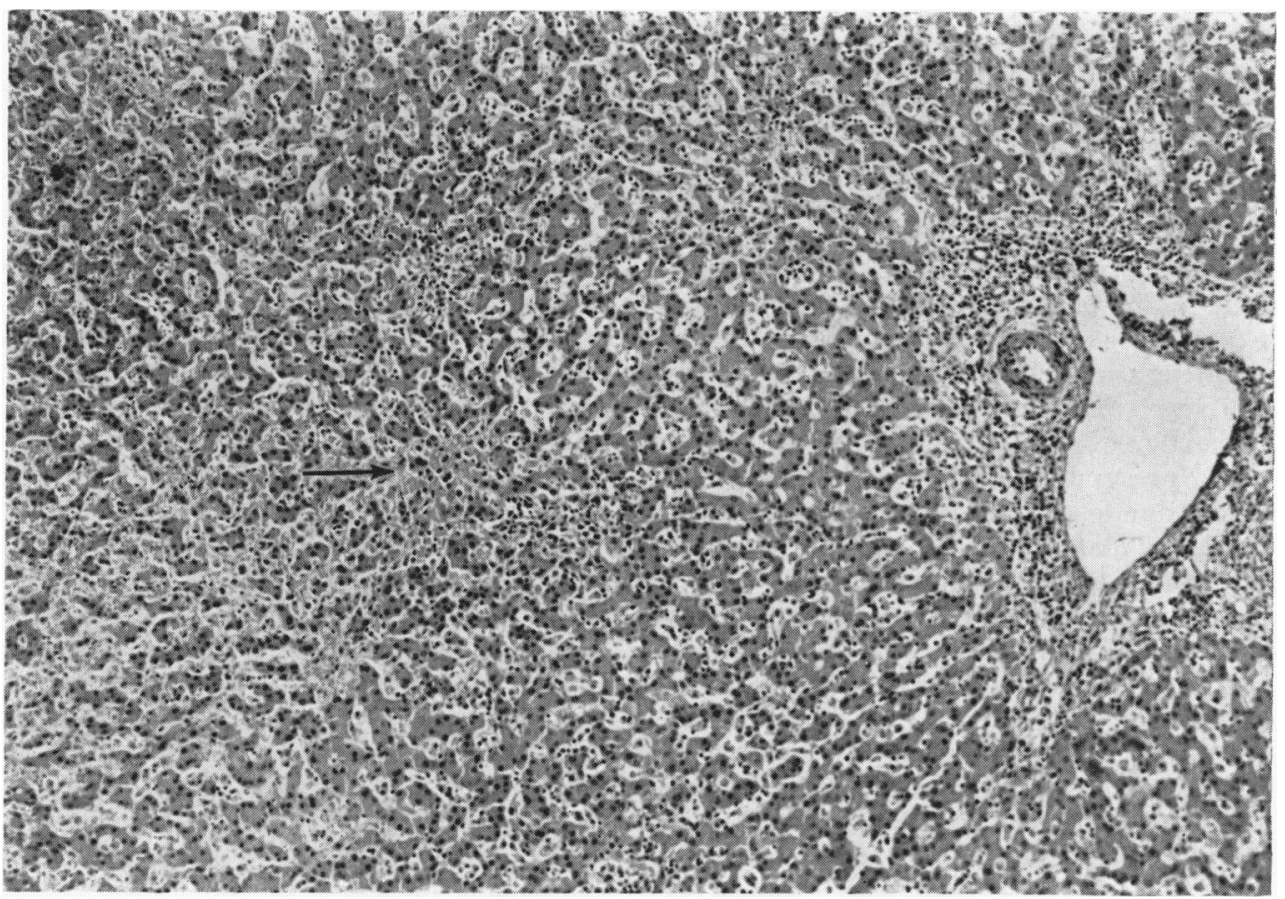

Fig. 3.-Case 2. Liver, showing many mononuclear cells in sinusoids with some portal infiltration. One area of early necrosis is seen (arrow). (H. and $E . \quad \times 25$. 


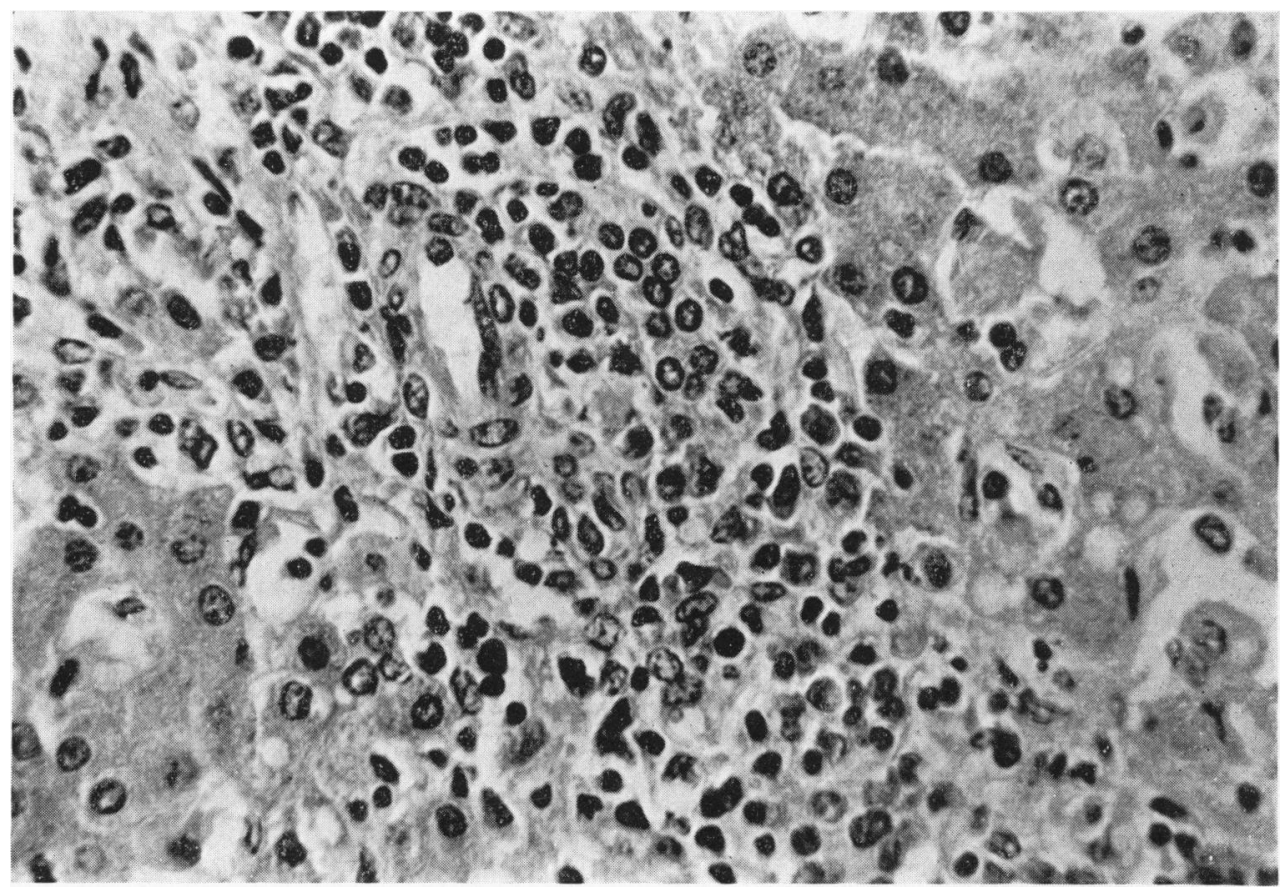

Fig. 4.-Case 2. Liver, showing mononuclear cell infiltration in portal area. (H. and $E . \quad \times 175$.

features were found at necropsy in both of our patients, and led to a diagnosis of acute leukaemia in the younger sister. Allen and Bass (1963) also considered this diagnosis in their fatal case, and other authors have commented on the similarity that may exist between these two diseases (Southam, Goldsmith, and Burchenal, 1951; Shinton and Hawkins, 1956). Southam et al. (1951) found heterophile antibody titres of more than $1: 56$ in $45 \%$ of cases of acute leukaemia. The antibodies were completely absorbed by guinea-pig kidney, and in this way the diagnosis of infectious mononucleosis can be excluded. The 2 children reported in this paper presumably died from the same infectious disease, due to an extremely virulent organism which presented many of the clinical and laboratory findings seen in infectious mononucleosis.

The causative agent is not established, but there is some evidence to show that this may be a virus and may be related to the myxovirus group (Joncas, Chagnon, and Pavilanis, 1966). The infectivity of the disease is low and, though it has been experimentally transmitted from man to animals, the results in human volunteers have been disappointing (Evans, 1960). The clinical features of the disease are shared by many other conditions. Mason and Adams (1958) suggest that the following criteria should be satisfied before the final diagnosis is made.
(1) Antibody titre of at least $1: 112$ without absorption.

(2) Following absorption with guinea-pig kidney, the titre to fall by at least $1 / 8$ th of the original titre.

(3) After beef absorption, the titre to fall to zero or near this.

(4) At least $50 \%$ of the total WBC to be lymphocytes.

(5) At least $15 \%$ of the lymphocytes to be atypical as described by Downey and McKinlay (1923).

Atypical lymphocytes, however, are not specific to infectious mononucleosis, for they may amount to more than $20 \%$ of the total WBC in infectious hepatitis, PAS, dilantoin and mesantoin hypersensitivity, and in lesser numbers in numerous other states (Wood and Frenkel, 1967). Case 1 fulfils these diagnostic criteria, and though no specific antibodies were found in Case 2, this does not exclude the diagnosis (Mason and Adams, 1958).

In the majority of patients, the liver is functionally and histologically abnormal. Rosalki, Jones, and Verney (1960) showed that the SGOT and SGPT was high in $83 \%$ of patients, and Mason and Adams (1958) demonstrated abnormal retention of bromsulphalein in $80 \%$ of non-jaundiced patients. Sullivan et al. (1957) found abnormal changes in the liver biopsies of 22 patients, and Hoagland and 
McCluskey (1955) and Nelson and Darragh (1956) found similar changes. There was inflammatory cell infiltration (predominantly lymphocytes and monocytes) in the portal tracts, in the sinusoids, and also within the lobule where they were randomly distributed as small foci of necrosis. The common occurrence of mild liver damage is usually short lived, and recovery is complete, though prolonged hepatitis may rarely follow (Cohn and Lidman, 1946). Sherlock (1963) does not consider that portal zone fibrosis or cirrhosis are sequelae of infectious mononucleosis.

Irreversible liver failure associated with infectious mononucleosis is extremely rare, and of the two reported patients, Allen and Bass (1963) describe a man aged 24 years who died 22 days after the onset of his illness; at necropsy the liver was enlarged and soft, and the cut surface was a deep golden yellow colour. Massive necrosis was present throughout the liver and some lobules were completely necrotic, while in others necrosis was most obvious in the more centrally placed cells. The portal tracts were infiltrated by abnormal mononuclear cells, lymphocytes, and neutrophils. Mononuclear cell infiltration was also found in the spleen, meninges, and kidneys, but the bone-marrow was normal.

The second patient was described by Ainley (1949). He died 10 days after the onset, and at necropsy the liver was found to be soft and pale yellow-brown in colour, with extensive centrilobular necrosis and portal tract infiltration.

\section{Summary}

Two sisters who died of infectious mononucleosis are described. In one, aged 22 months, the diagnosis was made before death, and she subsequently developed liver failure; in the other, aged 10 weeks, the diagnosis was retrospective, and she was originally thought to have died of acute leukaemia.

We are grateful to Sir Wilfrid Sheldon for permission to publish details of Case 1 and for his helpful advice. Dr. J. C. Valentine kindly provided information of the necropsy and histological findings in Case 2.

We thank Dr. C. Berry for reporting on the necropsy and histology in Case 1.

\section{REFERENCES}

Ainley, N. J. (1949). A fatal case of infectious mononucleosis with extensive zonal necrosis of the liver. Ulster med. F., 18, 219.

Allen, U. R., and Bass, B. H. (1963). Fatal hepatic necrosis in glandular fever. F. clin. Path., 16, 337.

Cohn, C., and Lidman, B. I. (1946). Hepatitis without jaundice in infectious mononucleosis. $\mathcal{F}$. clin. Invest., 25, 145.

Custer, R. P., and Smith, E. B. (1948). The pathology of infectious mononucleosis. Blood, 3, 830.

Downey, H., and McKinlay, C. A. (1923). Acute lymphadenosis compared with acute lymphatic leukemia. Arch. intern. Med., 32, 82.

Evans, A. S. (1960). Infectious mononucleosis in University of Wisconsin students. Amer. F. Hyg., 71, 342.

Hoagland, R. J., and McCluskey, R. T. (1955). Hepatitis in mononucleosis. Ann. intern. Med., 43, 1019.

Joncas, J., Chagnon, A., and Pavilanis, V. (1966). Studies on infectious mononucleosis. I. Cytopathic a zents and immunofluorescence studies. Canad. med. Ass. F., 94, 986.

Mason, W. R., Jr., and Adams, E. K. (1958). Infectious mononucleosis. An analysis of 100 cases with particular attention to diagnosis, liver function tests and treatment of selected cases with prednisone. Amer. F. med., Sci., 236, 447.

Nelson, R. S., and Darragh, J. H. (1956). Infectious mononucleosis hepatitis. Amer. F. Med., 21, 26.

Rosalki, S. B., Jones, T. G., and Verney, A. F. (1960). Transaminase and liver-function studies in infectious mononucleosis. Brit. med. F., 1, 929.

Sherlock, S. (1963). Diseases of the Liver and Biliary System. Blackwell, Oxford.

Shinton, N. K., and Hawkins, C. F. (1956). A fatal case of glandular fever. Lancet, 2, 708.

Southam, C. M., Goldsmith, Y., and Burchenal, J. H. (1951). Heterophile antibodies and antigens in neoplastic diseases. Cancer (Philad.), 4, 1036.

Sullivan, B. H., Jr., Irey, N. S., Pileggi, V. J., Crone, R. I., and Gibson, J. R. (1957). The liver in infectious mononucleosis. Amer. F. dig. Dis., 2, 210.

Wood, T. A., and Frenkel, E. P. (1967). The atypical lymphocyte. Amer. F. Med., 42, 923. 